\title{
Diversity of lichen flora of Odisha, India- A review
}

\author{
Nayak SK ${ }^{1}$, Bajpai $\mathbf{R}^{2}$, Upreti $\mathrm{DK}^{2}$ and Satapathy $\mathrm{KB}^{1 \&^{*}}$ \\ ${ }^{1}$ Post Graduate Department of Botany, Utkal University, Vani Vihar, Bhubaneswar, Odisha, India \\ ${ }^{2}$ Lichenology Laboratory, CSIR-National Botanical Research Laboratory, Lucknow, India. Email: \\ kbsbotuu@gmail.com
}

Nayak SK, Bajpai R, Upreti DK, Satapathy KB. 2016 - Diversity of lichen flora of Odisha, IndiaA review. Studies in Fungi 1(1), 114-124, Doi 10.5943/sif/1/1/11

\begin{abstract}
Based on the compilation of literature on lichens of Odisha (India) and other specimens and preserved in herbaria, we estimate that 252 species of lichens, belonging to 81 genera and 35 families are known from the state. Graphidaceae with 49 species is the dominant family, followed by Arthoniaceae (21), Pyrenulaceae (19), Parmeliaceae (18), Trypetheliaceae (17), Physciaceae (15), Teloschistaceae (14), Lecanoraceae (13), Pertusariaceae (12), Ramalinaceae (11), Caliciaceae (11), Thelotremataceae (9) and Lecidiaceae (4). Among the lichen genera Graphis is dominant with 27 species, followed by Pyrenula (16), Caloplaca (14), Pertusaria (12), Lecanora (10), Parmotrema (9), Cryptothecia (9) and Pyxine (6). Accounts of lichens are only available from 13 out of 30 districts. Of these only cursory collections from 10 districts are available, whereas Mayurbhanj, Jharsuguda and Ganjam districts are well-explored for their lichen flora. The present enumeration of more than 250 species clearly indicates a rich diversity of lichens for the state. Further exploration in other unexplored districts will add more species to the lichen flora of the state.
\end{abstract}

Key words - Ganjam - Jharsuguda - Mayurbhanj - mycobiont - phycobiont

\section{Introduction}

Lichens are the most fascinating and are widely distributed organisms on earth. These are the plants representing a unique symbiotic consortium comprising a phycobiont (alga) and a mycobiont (fungus) forming a thallus that does not resemble either symbionts in the free living (non-lichenized) state (Awasthi, 2000). Due to the peculiar structure and physiology, lichens are tolerant to extreme abiotic condition, such as drought or cold and they are able to grow in the diverse geographical regions from temperate to tropical in the Earth (Satya et al 2013). Lichens are the vital components of the ecosystem, in terms of substrata, shelter, food, nutrient cycles and succession (Gradstein, 1992). Lichens are found in a wide range of habitats all over the world and dominate terrestrial ecosystems. As many as 20,000 species of lichens have been reported worldwide. The Indian subcontinent is reportedly a reservoir of 2,450 species of lichens, of which India alone possesses more than 2300 species (Singh \& Sinha, 2010).

India as a mega-diversity country represents more than $10 \%$ of the total world species comprising about 2303 species under 305 genera and 74 families widely distributed in tropical, subtropical, temperate and alpine regions of India (Singh and Sinha, 2010). 
Odisha a coastal State at the eastern side of India located within the latitudes $17^{\circ} 78^{\prime} \mathrm{N} \&$ $22^{\circ} .73^{\prime} \mathrm{N}$ and longitudes $81^{\circ} .37^{\prime} \mathrm{E} \& 87^{\circ} .53^{\prime} \mathrm{E}$. Geographically the state is bounded by the states of West Bengal on the North East, Jharkhand, on the North, Chhatisgarh on the West, and the Andhra Pradesh on the South, the Bay of Bengal on the east. The state has costal line of about 450 $\mathrm{kms}$ and extends over an area of 155,707 sq. kms.

Singh and Sinha (2010) listed 44 species of lichens from the state of Odisha, India; However except enumeration of 35 species of lichens from Jharsuguda district (Upreti 1996), 13 species from hill forests of south Odisha (Vasundhara, 2008), 141 species species from Similipal Biosphere Reserve in Mayurbhanj district (Singh \& Kumar, 2012) and 12 species from Kapilash Reserve Forest in Dhenkanal district (Nayak et al, 2015) most regions have not been explored for their lichen species. The present review is prepared with an aim to understand the current status of distribution of lichens in different districts of the state. A clear idea of the diversity of lichens will be available once the hitherto unexplored or poorly explored areas are systematically studied for lichens.

\section{Materials \& Methods}

The review is based on published literature on lichens of Odisha and specimens lodged in CSIR-NBRI herbarium (LWG) from the state. Almost all research papers, monographs, project reports, abstracts available on lichens of the state of Odisha were thoroughly screened and lichen species mentioned therein were listed. Herbaria of CSIR-National Botanical Research Institute, Lucknow (LWG) and P.G. Department of Botany, Utkal University, Bhubaneswar were consulted to find out lichen specimens collected from Odisha in the past. The accession number, date, locality, name of collector- were noted down for compilation. A comprehensive list of lichen species occurring in Odisha was compiled with district-wise distribution together with nomenclatural changes of different taxa.

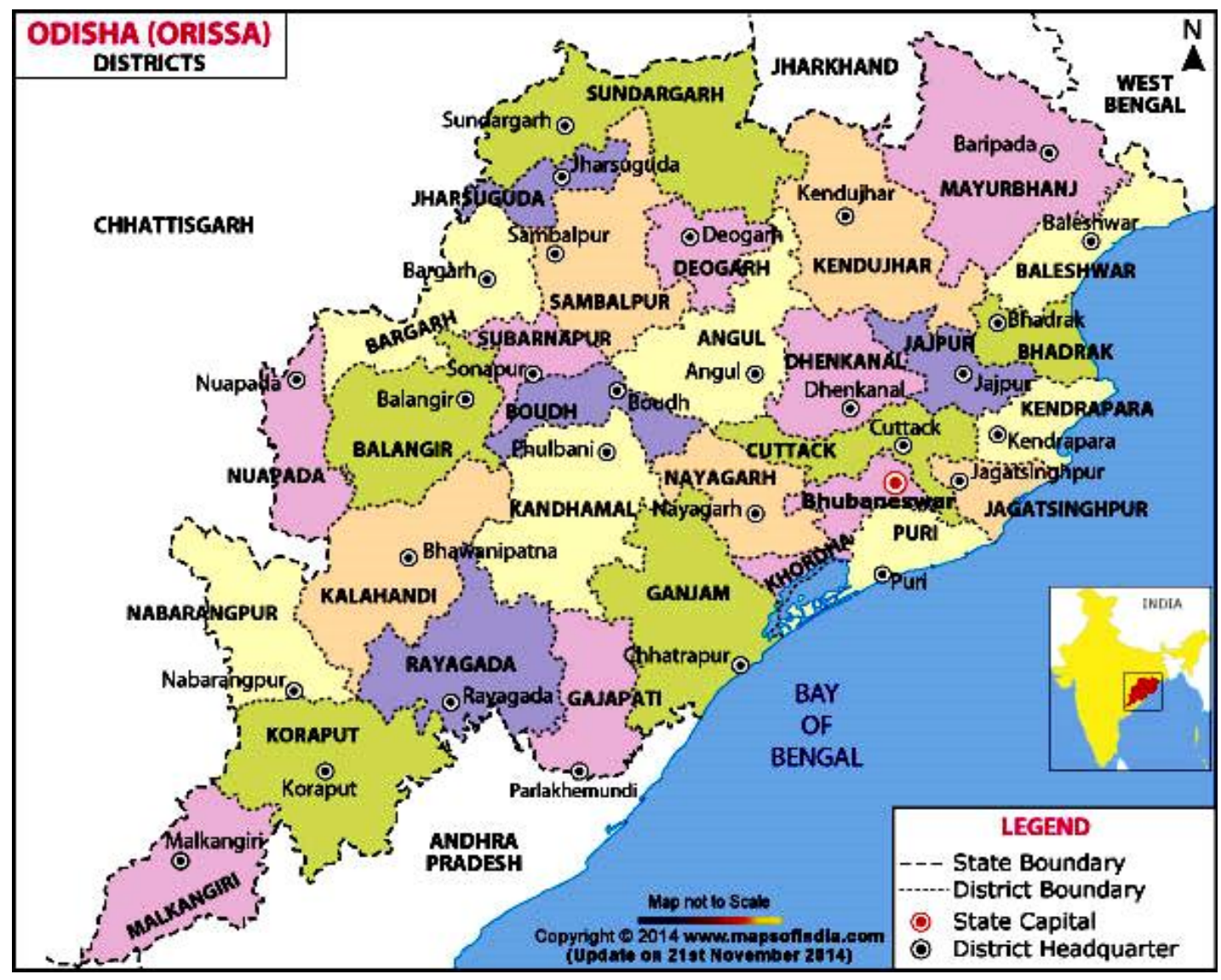

Fig.1 - Map of Odisha, India 


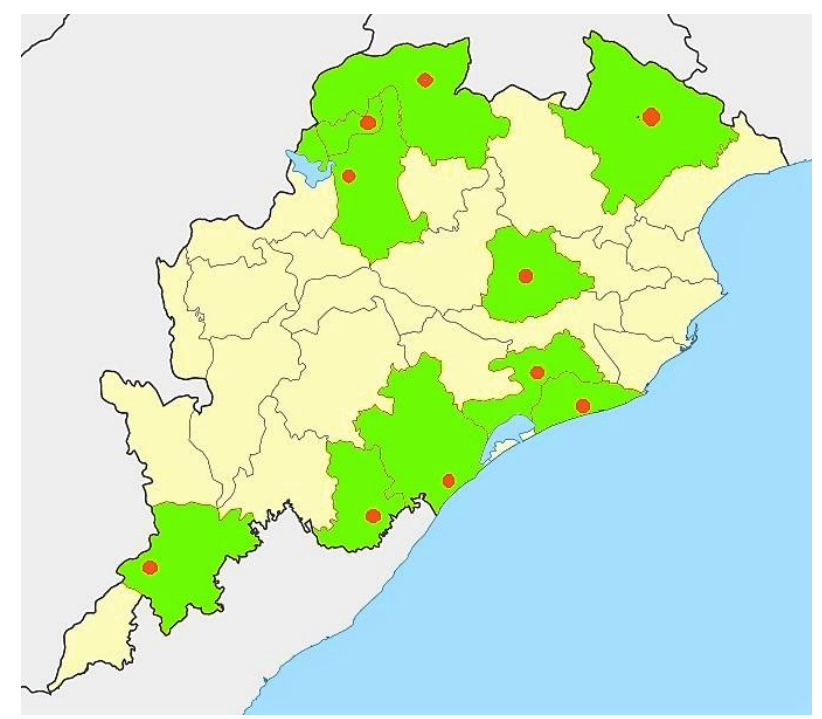

Fig. 2 - Sites from which lichen species are reported /collected in Odisha, India

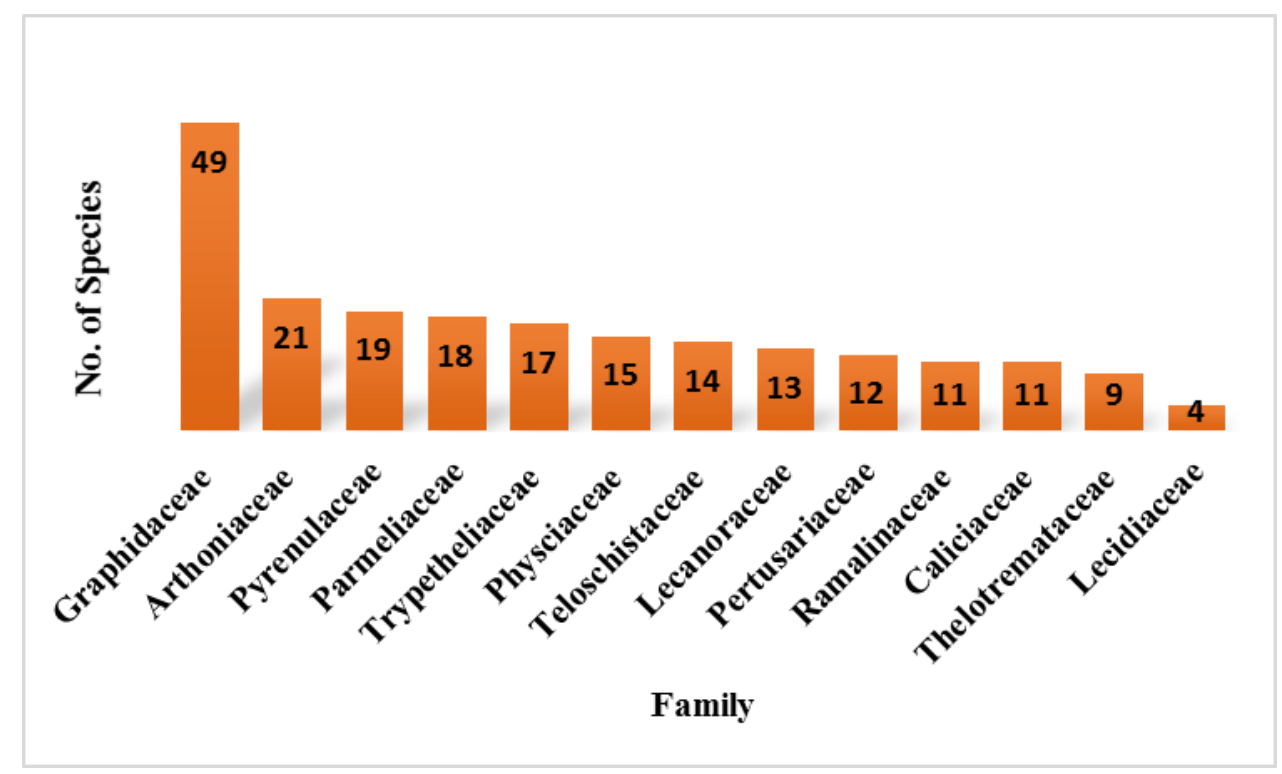

Fig. 3 - Family-wise distribution of lichen species in Odisha

\section{Results and Discussion}

The outcome of the work revealed the occurrence of 252 species of lichens belonging to 81 genera and 35 families in the state of Odisha (Table -1), Graphidaceae with 49 species is the dominant family followed by Arthoniaceae (21), Pyrenulaceae (19), Parmeliaceae (18), Trypetheliaceae (17), Physciaceae (15), Teloschistaceae (14), Lecanoraceae (12), Pertusariaceae (12), Ramalinaceae (11), Caliciaceae (11), Thelotremataceae (10) and Lecidiaceae (5). The lichen genus Graphis with 27 species dominates the state followed by Pyrenula (16), Caloplaca (14), Pertusaria (12), Lecanora (10), Parmotrema (9), Cryptothecia (9) and Pyxine (6). The Mayurbhanj district has the maximum diversity of lichens represented by141 species followed by Jharsuguda (31), Ganjam (21), Gajapati (7), Dhenkanal (5), Koraput (3), Khordha (3), Puri (1), Sambalpur (1) and Sundergarh (1). The distribution of lichens in different districts clearly indicates that only 10 districts were explored systematically for their lichen flora while the remaining 20 districts are completely unexplored for lichens. Occurrence of more than 250 species only form 10 districts indicates a rich diversity of lichens in the state. An extensive and intensive exploration of unexplored areas will definitely add many more lichen taxa to the lichen biota of the state. 
Table 1 Distribution of Lichens in different districts of Odisha, India

\begin{tabular}{|c|c|c|c|c|}
\hline Sl. No. & Name of Lichen & Family & Location & District/State \\
\hline 1. & $\begin{array}{l}\text { Anisomeridium terminatum (Nyl.) R.C. } \\
\text { Harris } 1995\end{array}$ & Monoblastaceae & Similipal & Mayurbhanj \\
\hline 2. & Anthracothecium pustuliferum Ajay Singh & Pyrenulaceae & - & Odisha \\
\hline 3. & $\begin{array}{l}\text { Anthracothecium thwaitesii (Leight) Müll. } \\
\text { Arg. }\end{array}$ & Pyrenulaceae & Similipal & Mayurbhanj \\
\hline 4. & $\begin{array}{l}\text { Anthracothecium variolosium (Pers.) } \\
\text { Müll.Arg. }\end{array}$ & Pyrenulaceae & Similipal & Mayurbhanj \\
\hline 5. & Arthonia medusula (Pers.) Nyl. & Arthoniaceae & Similipal & Mayurbhanj \\
\hline 6. & *Arthonia sp. & Arthoniaceae & Khordha & Khordha \\
\hline 7. & Arthonia translucens Stirt. & Arthoniaceae & Similipal & Mayurbhanj \\
\hline 8. & Arthonia tumidula (Ach.) Ach. & Arthoniaceae & Similipal & Mayurbhanj \\
\hline 9. & Arthopyrenia terminata (Nyl.) Mull. Arg. & Arthopyreniaceae & Kharubaba Ashram & Jharsuguda \\
\hline 10. & Arthothelium albescens Patw. \& Makhija & Arthoniaceae & Similipal & Mayurbhanj \\
\hline 11. & $\begin{array}{l}\text { Arthothelium confertum (A.L.Sm) Makhija \& } \\
\text { Patw. }\end{array}$ & Arthoniaceae & Similipal & Mayurbhanj \\
\hline 12. & Arthothelium erumpens Müll. Arg. & Arthoniaceae & Similipal & Mayurbhanj \\
\hline 13. & Arthothelium pycnocarpoides Müll. Arg. & Arthoniaceae & Similipal & Mayurbhanj \\
\hline 14. & *Aspicilia sp. & Megasporaceae & Seranga & Ganjam \\
\hline 15. & Bacidia alutacea (Kremp.) Zahlbr. & Ramalinaceae & Similipal & Mayurbhanj \\
\hline 16. & Bacidia convexula (Müll. Arg.) Zahlbr. & Ramalinaceae & Similipal & Mayurbhanj \\
\hline 17. & Bacidia medialis (Tuck.ex Nyl.) Zahlbr. & Ramalinaceae & Similipal & Mayurbhanj \\
\hline 18. & Bacidia millegrana (Taylor) Müll. Arg. & Ramalinaceae & Bagdihi & Jharsuguda \\
\hline 19. & Bacidia phaeolomoides (Müll. Arg.) Zahlbr. & Ramalinaceae & Similipal & Mayurbhanj \\
\hline 20. & $\begin{array}{l}\text { Bacidia psorina (Nyl. in Hue) Pant and } \\
\text { Awasthi }\end{array}$ & Ramalinaceae & Bagdihi & Jharsuguda \\
\hline 21. & *Bacidia sp. & Ramalinaceae & Mahendragiri & Ganjam \\
\hline 22. & Bacidia submedialis (Nyl.) Zahlbr. & Ramalinaceae & Bagdihi & Jharsuguda \\
\hline 23. & Bacidiospora psorina (Nyl.ex. Hue) Kalb. & Ramalinaceae & Similipal & Mayurbhanj \\
\hline 24. & Bathelium benghalense Müll. Arg. & Trypetheliaceae & Similipal & Mayurbhanj \\
\hline 25. & $\begin{array}{l}\text { Brigantiaea leucoxantha (Spreng.) R.Sant. \& } \\
\text { Hal.in Haf \& Bellem }\end{array}$ & Brigantiaceae & Belpahar & Jharsuguda \\
\hline 26. & Buellia alboatrior (Nyl.) Zahlbr & Caliciaceae & Similipal & Mayurbhanj \\
\hline 27. & $\begin{array}{l}\text { Buellia hemispherica S.R. Singh \& D.D. } \\
\text { Awasthi }\end{array}$ & Buelliaceae & - & Odisha \\
\hline 28. & *Buellia sp. & Caliciaceae & Gopalpur & Ganjam \\
\hline 29. & Buellia stellulata (Taylor) Mudd. & Caliciaceae & Similipal & Mayurbhanj \\
\hline 30. & Buellia tincta (Stein) Magnusson & Buelliaceae & Kharubaba Ashram & Jharsuguda \\
\hline 31. & Bulbothrix isidiza (Nyl.) Hale & Parmeliaceae & Similipal & Mayurbhanj \\
\hline 32. & Byssoloma tricholomum (Mont.) Zahlbr. & Pilocarpaceae & Similipal & Mayurbhanj \\
\hline 33. & Calopedia puiggarii (Müll. Arg.) Vezda & Ectolechiaceae & Similipal & Mayurbhanj \\
\hline 34. & Caloplaca aurantia (Pers.) Hellborn & Teloschistaceae & Similipal & Mayurbhanj \\
\hline 35. & Caloplaca bassiae (Willd.ex. Ach.) Zahlbr. & Teloschistaceae & Similipal & Mayurbhanj \\
\hline 36. & Caloplaca biatorina (A. Massal.) J. Steiner & Teloschistaceae & - & South Odisha \\
\hline 37. & $\begin{array}{l}\text { Caloplaca cinnabarina (Ach.) Zahlbr. in } \\
\text { Engl. \& Prantl }\end{array}$ & Teloschistaceae & - & Odisha \\
\hline
\end{tabular}




\begin{tabular}{|c|c|c|c|c|}
\hline Sl. No. & Name of Lichen & Family & Location & District/State \\
\hline 38. & Caloplaca cupulifera (Vain.) Zahlbr. & Teloschistaceae & - & Odisha \\
\hline 39. & Caloplaca encephalartii (Kremp) Zahlbr. & Teloschistaceae & Similipal & Mayurbhanj \\
\hline 40. & Caloplaca herbidella (Nyl.ex. Hue) Magn. & Teloschistaceae & Similipal & Mayurbhanj \\
\hline 41. & Caloplaca malaensis (Rasanen) Awasthi & Teloschistaceae & Bagdihi & Jharsuguda \\
\hline 42. & Caloplaca orissensis (Räsänen) D.D. Awasthi & Teloschistaceae & - & Odisha \\
\hline 43. & Caloplaca pollinii (A. Massal.) Jatta & Teloschistaceae & - & Odisha \\
\hline 44. & *Caloplaca sipeana Magn. & Teloschistaceae & Hirakud dam & Sambalpur \\
\hline 45. & *Caloplaca sp. & Teloschistaceae & Seranga & Ganjam \\
\hline 46. & Caloplaca subpoliotera Y. Joshi \& Upreti & Teloschistaceae & - & Odisha \\
\hline 47. & Caloplaca vitellinula (Nyl) H. Olivier & Teloschistaceae & Similipal & Mayurbhanj \\
\hline 48. & Cetraria melaloma Kremp. - Wei & Parmeliaceae & - & South Odisha \\
\hline 49. & Cetraria olivetorum Nyl. & Parmeliaceae & - & South Odisha \\
\hline 50. & Chapsa pseudophlyctic (Nyl.)A. Frisch & Theolotremataceae & Similipal & Mayurbhanj \\
\hline 51. & $\begin{array}{l}\text { *Cococarpia palmicola (Spreng.) Arvind \& } \\
\text { Gall }\end{array}$ & Coccocarpiaceae & Mahendragiri & Gajapati \\
\hline 52. & *Cococarpia pellita (Arch.) Mull.Arg.R.Sanl. & Coccocarpiaceae & Mahendragiri & Gajapati \\
\hline 53. & *Collema sp. & Collemataceae & Seranga & Ganjam \\
\hline 54. & Conotrema lumbricoides Sipman & Stictidaceae & Similipal & Mayurbhanj \\
\hline 55. & Cryptothecia multipuntata Jagdeesh \& al. & Arthoniaceae & Similipal & Mayurbhanj \\
\hline 56. & Cryptothecia bengalensis Jagadeesh \& al. & Arthoniaceae & Similipal & Mayurbhanj \\
\hline 57. & Cryptothecia effusa (Müll. Arg.) R. Sant. & Arthoniaceae & Similipal & Mayurbhanj \\
\hline 58. & Cryptothecia involuta Stirt. & Arthoniaceae & Similipal & Mayurbhanj \\
\hline 59. & $\begin{array}{l}\text { Cryptothecia lunulata (Zahlbr.) Makhija \& } \\
\text { Patw }\end{array}$ & Arthoniaceae & Kapilash & Dhenkanal \\
\hline 60. & Cryptothecia punctulata Makh. \& Patw. & Arthoniaceae & Kharubaba Ashram & Jharsuguda \\
\hline 61. & *Cryptothecia sp. & Arthoniaceae & Khordha & Khordha \\
\hline 62. & Cryptothecia subtecta Stirt. & Arthoniaceae & Similipal & Mayurbhanj \\
\hline 63. & $\begin{array}{l}\text { Cryptothecia phlyctidiformis (Müll. } \quad \text { Arg.) } \\
\text { D.D. Awasthi \& Kr.P. Singh }\end{array}$ & Arthoniaceae & Kharubaba Ashram & Jharsuguda \\
\hline 64. & Chrysothrix candelaris (L.) Laundon & Chrysothricaceae & Bagdihi & Jharsuguda \\
\hline 65. & *Dermatocarpon miniatum (L.) Mann. & Verrucariaceae & - & Ganjam \\
\hline 66. & Diorygma hieroglyphicum (Pers.) Kalb \& al. & Graphidaceae & Similipal & Mayurbhanj \\
\hline 67. & $\begin{array}{l}\text { Diorygma junghuhnii (Mont. \& Bosch.) Kalb. } \\
\& \text { al. }\end{array}$ & Graphidaceae & Similipal & Mayurbhanj \\
\hline 68. & Diorygma megasporrum Kalb. \& al. & Graphidaceae & Similipal & Mayurbhanj \\
\hline 69. & Diorygma pruinnosum (Eschw.) Kalb. Staiger & Graphidaceae & Similipal & Mayurbhanj \\
\hline 70. & $\begin{array}{l}\text { Diorygma radiatum (Awasthi \& S.R.Singh) } \\
\text { Kr. P. Singh \& Swarnalatha }\end{array}$ & Graphidaceae & Similipal & Mayurbhanj \\
\hline 71. & $\begin{array}{l}\text { *Diploschistes candidissimus (Kremp.) } \\
\text { Zahlbr. }\end{array}$ & Theolotremataceae & - & Ganjam \\
\hline 72. & $\begin{array}{l}\text { Diploschistes muscorum spp. bartelttii } \\
\text { Lumbsch. }\end{array}$ & Theolotremataceae & Similipal & Mayurbhanj \\
\hline 73. & Dirinaria aegialita (Afz. In Ach.) Moore & Physciaceae & Bagdihi & Jharsuguda \\
\hline 74. & Dirinaria applanata D.D. Awasthi. & Physciaceae & Similipal & Mayurbhanj \\
\hline 75. & *Dirinaria confluens (Fée) Awasthi & Physciaceae & - & Odisha \\
\hline 76. & Dirinaria consimilis (Stirt.) D.D. Awasthi. & Physciaceae & Similipal & Mayurbhanj \\
\hline 77. & *Dirinaria sp. & Physciaceae & - & Ganjam \\
\hline
\end{tabular}




\begin{tabular}{|c|c|c|c|c|}
\hline Sl. No. & Name of Lichen & Family & Location & District/State \\
\hline 78. & Drinaria papillulifera (Nyl.) D.D. Awasthi & Physciaceae & - & Odisha \\
\hline 79. & Dyplolabia afzelii (Ach.) A. Massal & Graphidaceae & Similipal & Mayurbhanj \\
\hline 80. & Glyphis cicatricosa Ach. & Graphidaceae & Similipal & Mayurbhanj \\
\hline 81. & Glyphis confluens Zenker & Graphidaceae & - & Odisha \\
\hline 82. & Glyphis duriuscula Stirt. & Graphidaceae & Similipal & Mayurbhanj \\
\hline 83. & Glyphis scyphulifera (Ach.) Staiger & Graphidaceae & Similipal & Mayurbhanj \\
\hline 84. & Graphis caesiella Vain & Graphidaceae & Similipal & Mayurbhanj \\
\hline 85. & Graphis cincta (Pers) Aptroot & Graphidaceae & Similipal & Mayurbhanj \\
\hline 86. & Graphis distincta Makhija \& Adaw & Graphidaceae & Similipal & Mayurbhanj \\
\hline 87. & Graphis furcata Fee & Graphidaceae & Similipal & Mayurbhanj \\
\hline 88. & Graphis glaucescence Fee & Graphidaceae & Similipal & Mayurbhanj \\
\hline 89. & Graphis hiascens (Fee) Archer & Graphidaceae & Similipal & Mayurbhanj \\
\hline 90. & Graphis japonica (Mull. Arg) A.W. Archer & Graphidaceae & Similipal & Mayurbhanj \\
\hline 91. & Graphis agarekarii Patw. \& C.R.Kulk. & Graphidaceae & Similipal & Mayurbhanj \\
\hline 92. & Graphis albidofarinaceae Adaw. \& Makhija & Graphidaceae & Similipal & Mayurbhanj \\
\hline 93. & Graphis albissima Müll. Arg & Graphidaceae & Similipal & Mayurbhanj \\
\hline 94. & Graphis chloroalba Makhija \& Adaw. & Graphidaceae & Similipal & Mayurbhanj \\
\hline 95. & *Graphis elegans (Borrer ex Sm.) Ach. & Graphidaceae & - & Ganjam \\
\hline 96. & Graphis filiformis Adaw. \& Makhija & Graphidaceae & Similipal & Mayurbhanj \\
\hline 97. & Graphis garoana Nagarkar \& Patw. & Graphidaceae & - & Odisha \\
\hline 98. & Graphis handelii Zahlbr & Graphidaceae & Similipal & Mayurbhanj \\
\hline 99. & Graphis insulana (Mull. Arg) Lucking & Graphidaceae & Similipal & Mayurbhanj \\
\hline 100. & Graphis intermediella Stirt. & Graphidaceae & - & Odisha \\
\hline 101. & Graphis librata C. Knight & Graphidaceae & Similipal & Mayurbhanj \\
\hline 102. & *Graphis nakanishiana Patw. \& Kaul. & Graphidaceae & - & Jharsuguda \\
\hline 103. & Graphis perticosa (Kremp.) A.W. Archer & Graphidaceae & Similipal & Mayurbhanj \\
\hline 104. & Graphis pinicola Zahlbr & Graphidaceae & Similipal & Mayurbhanj \\
\hline 105. & Graphis platycarpa Eschw & Graphidaceae & Similipal & Mayurbhanj \\
\hline 106. & Graphis pyrrhocheiloides Zahlbr & Graphidaceae & Similipal & Mayurbhanj \\
\hline 107. & Graphis scripta (L.) Ach. & Graphidaceae & Similipal & Mayurbhanj \\
\hline 108. & Graphis streblocarpa (Bel.) Nyl. & Graphidaceae & Similipal & Mayurbhanj \\
\hline 109. & Graphis subasahinae Nagarkar \& Patw. & Graphidaceae & Similipal & Mayurbhanj \\
\hline 110. & Graphis tenella Ach. & Graphidaceae & Similipal & Mayurbhanj \\
\hline 111. & *Haematomma sp. & Haematommataceae & Seranga & Ganjam \\
\hline 112. & Haematomma wattii (Stirt.) Zahlbr. & Haematommataceae & Similipal & Mayurbhanj \\
\hline 113. & Hafellia curatellae (Malme) Marbach & Caliciaceae & Similipal & Mayurbhanj \\
\hline 114. & *Heppia sp. & Heppiaceae & Barkuda & Ganjam \\
\hline 115. & Herpothallon isidiatum Jagdeesh \& al & Arthoniaceae & Similipal & Mayurbhanj \\
\hline 116. & Heterodermia pseudospeciosa (Kurok) Culb & Physciaceae & Similipal & Mayurbhanj \\
\hline 117. & $\begin{array}{l}\text { Heterodermia diademata (Taylor) D.D. } \\
\text { Awasthi }\end{array}$ & Physciaceae & - & South Odisha \\
\hline 118. & Heterodermia obscurata (Nyl.) Trevis & Physciaceae & Similipal & Mayurbhanj \\
\hline 119. & *Heterodermia sp. & Physciaceae & Mahendragiri & Gajapati \\
\hline 120. & Heterodermia speciosa (Wulfen) Trevis & Physciaceae & Kapilash & Dhenkanal \\
\hline 121. & $\begin{array}{l}\text { Heterodermia albidiflava (Kurok.) D.D. } \\
\text { Awasthi }\end{array}$ & Physciaceae & Similipal & Mayurbhanj \\
\hline
\end{tabular}




\begin{tabular}{|c|c|c|c|c|}
\hline Sl. No. & Name of Lichen & Family & Location & District/ State \\
\hline 122. & $\begin{array}{l}\text { Laurera tuberculosum Makhija \& Patw } \\
\text { Harris }\end{array}$ & Trypetheliaceae & Similipal & Mayurbhanj \\
\hline 123. & Laurera cumingii (Mont) Zahlbr. & Trypetheliaceae & Similipal & Mayurbhanj \\
\hline 124. & Laurera keralensis Upreti \& A. Singh & Trypetheliaceae & Similipal & Mayurbhanj \\
\hline 125. & Laurera kundarensis Upreti \&A.Singh & Trypetheliaceae & Similipal & Mayurbhanj \\
\hline 126. & Laurera vezdae Makhija \& Patw & Trypetheliaceae & Similipal & Mayurbhanj \\
\hline 127. & Laurera aurantiaca Makhija \& Patw. & Trypetheliaceae & Similipal & Mayurbhanj \\
\hline 128. & *Lecanora sp. & Lecanoraceae & Bhubaneswar & Khordha \\
\hline 129. & Lecanora argentata (Ach.) Digel.in Nilsson & Lecanoraceae & - & Odisha \\
\hline 130. & $\begin{array}{l}\text { Lecanora cinerofusca var. cinerofusca } \\
\text { H.Magn }\end{array}$ & Lecanoraceae & Similipal & Mayurbhanj \\
\hline 131. & *Lecanora helva Stizenb. 1890 & Lecanoraceae & Jharsuguda & Jharsuguda \\
\hline 132. & Lecanora iseana Räsänen & Lecanoraceae & Similipal & Mayurbhanj \\
\hline 133. & Lecanora perplexa Brodo & Lecanoraceae & - & Odisha \\
\hline 134. & Lecanora pulicaris (Pers.) Ach. & Lecanoraceae & Bagdihi & Jharsuguda \\
\hline 135. & Lecanora rugosella Zahlbr. & Lecanoraceae & - & Odisha \\
\hline 136. & Lecanora subimmersa (Fée) Vain. & Lecanoraceae & - & Odisha \\
\hline 137. & Lecanora tropica Zahlbr, & Lecanoraceae & - & Odisha \\
\hline 138. & Lecidea lapicida (Ach.) Ach. & Lecideaceae & Similipal & Mayurbhanj \\
\hline 139. & Lecidea plana (J. Lahm.) Nyl. & Lecideaceae & Similipal & Mayurbhanj \\
\hline 140. & Lecidea secernes H. Magn & Lecideaceae & Similipal & Mayurbhanj \\
\hline 141. & Lecidea sp. & Lecidiaceae & Bagdihi & Jharsuguda \\
\hline 142. & Lecidella enteroleucella ( $\mathrm{Nyl}$.$) Hertel$ & Lecanoraceae & Similipal & Mayurbhanj \\
\hline 143. & $\begin{array}{l}\text { Leiorruma exaltatum (Mont. \&V.D. Bosch) } \\
\text { Müll. Arg. }\end{array}$ & Graphidaceae & Similipal & Mayurbhanj \\
\hline 144. & *Lepraria sp. 1 & Stereocaulaceae & Jharsuguda & Jharsuguda \\
\hline 145. & Lepraria lobificans Nyl. & Stereocaulaceae & Kapilash & Dhenkanal \\
\hline 146. & *Lepraria sp. 2 & Stereocaulaceae & - & Odisha \\
\hline 147. & $\begin{array}{l}\text { Leptogium austro-americanum (Malme) } \\
\text { Dodge }\end{array}$ & Parmeliaceae & Bagdihi & Jharsuguda \\
\hline 148. & $*$ Leptogium sp. & Collemataceae & Chandragiri & Gajapati \\
\hline 149. & Leptogium trichophorum Müll. Arg. - Wei & Thelotremataceae & - & South Odisha \\
\hline 150. & Letrouitia leprolyta (Nyl.) Hafellner & Letrouitiaceae & Similipal & Mayurbhanj \\
\hline 151. & Letrouitia domingenesis Hafellner \& Bellem & Letrouitiaceae & Similipal & Mayurbhanj \\
\hline 152. & *Letrouitia sp. & Letrouitiaceae & Mahendragiri & Gajapati \\
\hline 153. & $\begin{array}{l}\text { Letrouitia transgrassa (Malme) Hafellner \& } \\
\text { Bellem }\end{array}$ & Letrouitiaceae & Similipal & Mayurbhanj \\
\hline 154. & Lobaria pulmonaria (L.) Hoffm. & Lobariaceae & - & South Odisha \\
\hline 155. & $\begin{array}{l}\text { *Malmidea granifera (Ach.) Kalb, Rivas Plata } \\
\& \text { Lumbsch }\end{array}$ & Pilocarpaceae & - & $\begin{array}{l}\text { Ganjam } \\
\text { Mayurbhanj }\end{array}$ \\
\hline 156. & Megalotremis biocellata Aptroot & Trypetheliaceae & Similipal & Mayurbhanj \\
\hline 157. & $\begin{array}{l}\text { Mycomicrothelia conothelina (Nyl.) } \\
\text { Hawksw. }\end{array}$ & Arthopyreniaceae & Similipal & Mayurbhanj \\
\hline 158. & $\begin{array}{l}\text { Myelochroa xantholepis (Mant. \& Bosch.) } \\
\text { Elix }\end{array}$ & Parmeliaceae & Similipal & Mayurbhanj \\
\hline 159. & $\begin{array}{l}\text { *Myriotrema norstictideum (Patw. \& } \\
\text { Nagarkar) D.D. Awasthi }\end{array}$ & Theolotremataceae & Similipal & Mayurbhanj \\
\hline
\end{tabular}




\begin{tabular}{|c|c|c|c|c|}
\hline Sl. No. & Name of Lichen & Family & Location & District/ State \\
\hline 160 & Ocellularia lankaensis Hale & Theolotremataceae & Similipal & Mayurbhanj \\
\hline 161. & Opegrapha rufescens Pers & Rocellaceae & Similipal & Mayurbhanj \\
\hline 162. & Opegrapha sp. & Opegraphaceae & Kharubaba Ashram & Jharsuguda \\
\hline 163. & $\begin{array}{l}\text { Pallidogramme chrysenteron (Mont.) Staiger } \\
\& \text { al. }\end{array}$ & Graphidaceae & Similipal & Mayurbhanj \\
\hline 164. & Parmelia awasthii (Hale \& Patw) Awasthi & Parmeliaceae & Belpahar & Jharsuguda \\
\hline 165. & Parmelia mesotropa Müll. Arg & Parmeliaceae & Bagdihi & Jharsuguda \\
\hline 166. & *Parmelia sp. & Parmeliaceae & Chandragiri & Gajapati \\
\hline 167. & Parmeliella papillata P.M. Jørg. & Parmeliaceae & - & Odisha \\
\hline 168. & Parmotrema andium (Müll. Arg.) Hale & Parmeliaceae & Similipal & Mayurbhanj \\
\hline 169. & Parmotrema austrosinenese (Zahlbr) Hale & Parmeliaceae & Kapilash & Dhenkanal \\
\hline 170. & Parmotrema cristiferum (Taylor) Hale & Parmeliaceae & - & Odisha \\
\hline 171. & Parmotrema hababianum (Gyeln.) Hale. & Parmeliaceae & - & Odisha \\
\hline 172. & Parmotrema indicum Hale & Parmeliaceae & - & Odisha \\
\hline 173. & Parmotrema kamatii Patw. \& A.V. Prabhu & Parmeliaceae & - & Odisha \\
\hline 174. & $\begin{array}{l}\text { Parmotrema ravum (Krog \& Swinscow) } \\
\text { Sèrus }\end{array}$ & Parmeliaceae & Similipal & Mayurbhanj \\
\hline 175. & Parmotrema reticulatum (Taylor) M. Choisy & Parmeliaceae & Kapilash & Dhenkanal \\
\hline 176. & Parmotrema tinctorum (Despr. ex. Nyl.) Hale & Parmeliaceae & Similipal & Mayurbhanj \\
\hline 177. & $\begin{array}{l}\text { Pertusaria albescens (Huds.) Choisy \&Wern. } \\
\text { in Wem. }\end{array}$ & Pertusariaceae & Bagdihi & Jharsuguda \\
\hline 178. & Pertusaria cinchonae Müll. Arg & Pertusariaceae & Bagdihi & Jharsuguda \\
\hline 179. & Pertusaria kodaikanalensis Choisy & Pertusariaceae & Similipal & Mayurbhanj \\
\hline 180. & Pertusaria leioplacella $\mathrm{Nyl}$. & Pertusariaceae & - & Odisha \\
\hline 181. & *Pertusaria leucosora $\mathrm{Nyl}$ & Pertusariaceae & Raisili & Koraput \\
\hline 182. & Pertusaria leucostoma (Bernh.) A. Massal. & Pertusariaceae & - & Odisha \\
\hline 183. & Pertusaria melastomela $\mathrm{Nyl}$. & Pertusariaceae & Similipal & Mayurbhanj \\
\hline 184. & Pertusaria multipuncta (Turner) Nyl & Pertusariaceae & Similipal & Mayurbhanj \\
\hline 185. & Pertusaria pertusa (Weigel) Tuck. & Pertusariaceae & Bagdihi & Jharsuguda \\
\hline 186. & Pertusaria quassiae (Fẻe) Nyl. & Pertusariaceae & Similipal & Mayurbhanj \\
\hline 187. & *Pertusaria sp. & Pertusariaceae & Raisili & Koraput \\
\hline 188. & Pertusaria concinna Erichsen & Pertusariaceae & Bagdihi & Jharsuguda \\
\hline 189. & Phaeographina limbata Müll. Arg & Graphidaceae & Kharubaba Ashram & Jharsuguda \\
\hline 190. & $\begin{array}{l}\text { Phaeographina pudica var platyloma } \\
\text { (Müll.) Arg. Redinger }\end{array}$ & Graphidaceae & Kharubaba Ashram & Jharsuguda \\
\hline 191. & $\begin{array}{l}\text { Phaeographina caesiopruinosa. (Fée) Müll. } \\
\text { Arg }\end{array}$ & Graphidaceae & Kharubaba Ashram & Jharsuguda \\
\hline 192. & $\begin{array}{l}\text { Phaeographis brasiliensis (A.Massal.) Kalb. } \\
\text { \& Matthes-Leicht }\end{array}$ & Graphidaceae & Similipal & Mayurbhanj \\
\hline 193. & *Pheographis sp. & Graphidaceae & Berhampur & Ganjam \\
\hline 194. & *Physcia phaea (Tuck) Thomson & Physciaceae & Belpahar & Jharsuguda \\
\hline 195. & Physcia cylindrophora (Taylor) Nyl. & Physciaceae & Bagdihi & Jharsuguda \\
\hline 196. & $\begin{array}{l}\text { Platygramme platyloma (Müll. Arg.) M. } \\
\text { Nakan. \& Kashiw. }\end{array}$ & Graphidaceae & - & Odisha \\
\hline 197. & $\begin{array}{l}\text { *Platygramme pudica (Ment. Etv.d.Basch) } \\
\text { Zahlber }\end{array}$ & Graphidaceae & Jharsuguda & Jharsuguda \\
\hline 198. & Platythecium grammitis (Fée) Staiger & Graphidaceae & Similipal & Mayurbhanj \\
\hline
\end{tabular}




\begin{tabular}{|c|c|c|c|c|}
\hline Sl. No. & Name of Lichen & Family & Location & District/ State \\
\hline 199. & Pleurotrema filisporum Patw.Makh. \& Ranie & Physciaceae & Sundergarh & Sundergarh \\
\hline 200. & Polymeridium proponens (Nyl.) R.C. Harris & Trypetheliaceae & Similipal & Mayurbhanj \\
\hline 201. & Porina tetracerae (Afz.) Müll. Arg. in Engler & Porinaceae & - & Odisha \\
\hline 202. & Pseudopyrenula pupula (Ach.) Müll. Arg. & Trypetheliaceae & Similipal & Mayurbhanj \\
\hline 203. & Pseudopyrenula subnudata Müll. Arg. & Trypetheliaceae & Similipal & Mayurbhanj \\
\hline 204. & Pseudopyrenula subvelata Müll. Arg. & Trypetheliaceae & Similipal & Mayurbhanj \\
\hline 205. & Pyrenula acutalis R.C Harris & Pyrenulaceae & Similipal & Mayurbhanj \\
\hline 206. & Pyrenula anomela (Ach.) Vain. & Pyrenulaceae & Similipal & Mayurbhanj \\
\hline 207. & Pyrenula brunnea Vainio & Pyrenulaceae & Kharubaba Ashram & Jharsuguda \\
\hline 208. & Pyrenula citriformis R.C. Harris & Pyrenulaceae & Similipal & Mayurbhanj \\
\hline 209. & Pyrenula introducta (Nyl.) Zahlr. & Pyrenulaceae & Similipal & Mayurbhanj \\
\hline 210. & Pyrenula kurzii Ajay Singh \& Upreti & Pyrenulaceae & - & Odisha \\
\hline 211. & Pyrenula leucotrypa (Nyl.) Upreti. & Pyrenulaceae & Similipal & Mayurbhanj \\
\hline 212. & Pyrenula macularis (Zahlbr.) R.C. Harris & Pyrenulaceae & - & Odisha \\
\hline 213. & Pyrenula mamillana (Ach.) Trevis. & Pyrenulaceae & Similipal & Mayurbhanj \\
\hline 214. & Pyrenula nitens Fẻe & Pyrenulaceae & Similipal & Mayurbhanj \\
\hline 215. & Pyrenula oculiferaVain. & Pyrenulaceae & - & Odisha \\
\hline 216. & *Pyrenula sp. & Pyrenulaceae & Seranga & Ganjam \\
\hline 217. & Pyrenula subacutalis Upreti & Pyrenulaceae & - & Odisha \\
\hline 218 & $\begin{array}{l}\text { Pyrenula sublaevigata (Patw. \& Makhija) } \\
\text { Upreti }\end{array}$ & Pyrenulaceae & Similipal & Mayurbhanj \\
\hline 219. & Pyrenula subnitida (Nyl.) Müll. Arg. & Pyrenulaceae & Similipal & Mayurbhanj \\
\hline 220. & Pyrenula thelomopha Tuck. & Pyrenulaceae & Similipal & Mayurbhanj \\
\hline 221. & *Pyxine berteriana $(\mathrm{Fee}) \mathrm{Fw}$ & Caliciaceae & Jeypore & Koraput \\
\hline 222. & Pyxine coccifera (Fẻe) Nyl. & Physciaceae & Similipal & Mayurbhanj \\
\hline 223. & Pyxine cocoes (Sw.) Nyl. & Caliciaceae & - & Odisha \\
\hline 224. & *Pyxine consocians Vain & Caliciaceae & Barkuda & Ganjam \\
\hline 225. & *Pyxine sp. & Caliciaceae & Taptapani & Ganjam \\
\hline 226. & *Pyxine subcinerea Strit. & Caliciaceae & -- & Ganjam \\
\hline 227. & Ramalina leiodea (Nyl.) Nyl. & Ramalinaceae & - & Odisha \\
\hline 228. & $*$ Ramalina sp. & Ramalinaceae & Taptapani & Ganjam \\
\hline 229. & $\begin{array}{l}\text { Ramboldia russula (Ach.) Kalb., Lumbsch \& } \\
\text { Elix }\end{array}$ & Lecanoraceae & Similipal & Mayurbhanj \\
\hline 230. & Ramboldia stuartii (Hampe) Kantvilas \& Elix & Lecanoraceae & - & Odisha \\
\hline 231. & Reimnitzia sentensis (Tuck.) Kalb. & Theolotremataceae & Similipal & Mayurbhanj \\
\hline 232. & Rinodina oxydata (A. Massal.) Massal. & Physciaceae & Similipal & Mayurbhanj \\
\hline 233. & *Roccella montagnei Bél. & Roccellaceae & Berhampur & Ganjam \\
\hline 234. & *Roccella sp. & Roccellaceae & Konark temple & Puri \\
\hline 235. & Sarcographa tricosa (Ach.) Mull. Arg. & Graphidaceae & Similipal & Mayurbhanj \\
\hline 236. & Schistophoron tenue Stirt. & Graphidaceae & - & Odisha \\
\hline 237. & $\begin{array}{l}\text { Sporopodium argillaceum (Müll. Arg.) } \\
\text { Zahlbr. }\end{array}$ & Ectolechiaceae & Similipal & Mayurbhanj \\
\hline 238. & Staurothele drummondii (Tuck.) Tuck. & Verrucariaceae & Similipal & Mayurbhanj \\
\hline 239. & Stereocaulon sp. & Steriocaulaceae & - & South Odisha \\
\hline 240. & Sticta praetextata (Ras.) D. D. Awasthi & Lobariaceae & - & South Odisha \\
\hline 241. & Strigula complanata ( Fée) Mont. & Strigulaceae & Similipal & Mayurbhanj \\
\hline 242. & Thelotrema albo-olivaceum Vain. & Theolotremataceae & Similipal & Mayurbhanj \\
\hline 243. & *Thelotrema leucocheilum & Theolotremataceae & Taptapani & Ganjam \\
\hline 244. & Trypethelium eluterae Spreng & Trypetheliaceae & Similipal & Mayurbhanj \\
\hline 245. & $\begin{array}{l}\text { Trypethelium endosulphureum Makhija \& } \\
\text { Patw. }\end{array}$ & Trypetheliaceae & Similipal & Mayurbhanj \\
\hline
\end{tabular}




\begin{tabular}{cllll}
\hline S1. No. & Name of Lichen & Family & Location & District/ State \\
\hline 246. & Trypethelium platystomum Mont. & Trypetheliaceae & Similipal & Mayurbhanj \\
247. & Trypethelium tropicum (Ach.) Müll. Arg. & Trypetheliaceae & Similipal & Mayurbhanj \\
248. & $*$ Trypthelium sp. & Trypetheliaceae & - & Ganjam \\
249. & Tylophoron moderatum Nyl. & Arthoniaceae & - & Odisha \\
250. & Tylophoron protrudens Nyl. & Arthoniaceae & Similipal & Mayurbhanj \\
251. & $*$ Tylophoron sp. & Arthoniaceae & Chandragiri & Gajapati \\
252. & Verrucaria rupestris Schrad & Verrucariaceae & Similipal & Mayurbhanj \\
\hline
\end{tabular}

* Collected from Odisha and deposited in Herbarium of CSIR- National Botanical Research Institute, Lucknow (LWG), India.

Table 2 Doubtful taxa with possible nomenclature

\begin{tabular}{|c|c|c|}
\hline Sl. No. & Doubtful taxa & Possible nomenclature \\
\hline 1. & Anthracothecium pustuliferum Ajay Singh & Pyrenula duplicans (Nyl.) Aptroot \\
\hline 2. & Anthracothecium thwaitesii (Leight) Müll. Arg. & Pyrenula papillifera (Nyl.) Aptroot \\
\hline 3. & Anthracothecium variolosium (Pers.) Müll.Arg. & Pyrenula globifera (Eschw.) Aptroot \\
\hline 4. & Arthopyrenia terminata (Nyl.) Mull. Arg. & Anisomeridium terminatum (Nyl.) R.C. Harris \\
\hline 5. & Laurera tuberculosum Makhija \& Patw Harris & Bathelium tuberculosum (Makhija \& Patw.) R.C. Harris \\
\hline 6. & Bathelium benghalense Müll.Arg. & $\begin{array}{l}\text { Marcelaria benguelensis (Müll. Arg.) Aptroot, Nelsen \& } \\
\text { Parnmen }\end{array}$ \\
\hline 7. & Graphis nakanishiana Patw. \& Kaul. & $\begin{array}{l}\text { Hemithecium nakanishianum (Patw. \& C.R. Kulk.) } \\
\text { Makhija \& Dube }\end{array}$ \\
\hline 8. & Pleurotrema filisporum Patw.Makh. \& Ranie & $\begin{array}{l}\text { Lithothelium filisporum (Patw., Makhija \& D. Rane) Kr.P. } \\
\text { Singh \& G.P. Sinha }\end{array}$ \\
\hline 9. & Pyrenula macularis (Zahlbr.) R.C. Harris & Pyrenula breutelii (Müll. Arg.) Aptroot, \\
\hline 10. & Sticta praetextata (Ras.) D. D. Awasthi & $\begin{array}{l}\text { Dendriscosticta praetextata (Räsänen) Moncada \& } \\
\text { Lücking }\end{array}$ \\
\hline
\end{tabular}

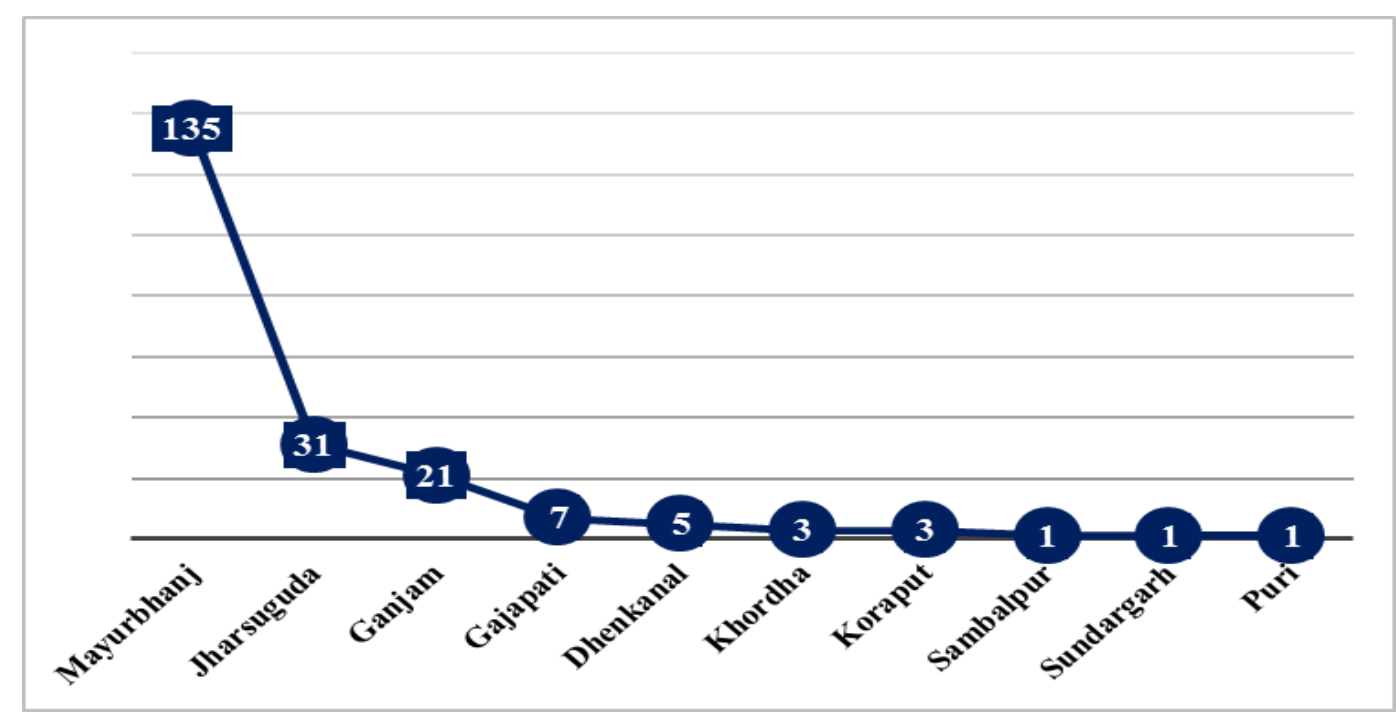

Fig. 4 - District-wise distribution of reported lichen species in Odisha 


\section{References}

Awasthi DD. 2000 - Lichenology in Indian Subcontinent. Bishen Singh Mahendra Pal Singh, Dehra Dun, India.

Gradstein SR. 1992 - The vanishing tropical rain as an environment for bryophytes and lichens. Pages 234-253, In: Bates, J.W and Farmer, A.M. (Editors) Bryophytes and Lichen in changing environment. Clarendon Press, Oxford.

Nayak SK, Mohapatra A, Chand PK and Satapathy KB. 2015 - Diversity of lichen flora in Kapilash reserve forest of Dhenkanal district in Odisha, India. Abstract presented in National conference on cryptogam research in India: Progress and Prospects, Indian lichenological society, Lucknow.

Report on Biodiversity assessment in some selected hill forests of south Orissa, Vasundhara, Bhubaneswar, Odisha, 2008

Satya, Upreti DK and Tandon PK. 2013 - Species diversity and host specificity of lichens: a case study in two forest stands of central India. International Journal of Ecology and Environmental Sciences 39 (4), 251-270.

Singh KP and Kumar K. 2012 - A note on the lichens from Similipal biosphere reserve, Indian Journal of Forestry, 35(3), 383-390.

Singh KP and Sinha GP. 2010 -Indian Lichens: Annotated Checklist. Botanical Survey of India, Kolkata

Upreti DK. 1996 - Lichen on Shorea robusta in Jharsuguda district of Orissa, India, Flora and Fauna 2(2), 159-161. 